\title{
High power Cherenkov radiation from a relativistic particle rotating around a dielectric ball
}

\author{
L.Sh. Grigoryan* H.F. Khachatryan, S.R. Arzumanyan† M.L. Grigoryan \\ Institute of Applied Problems in Physics \\ $25 \mathrm{Hr}$. Nersessian Str., 375014 Yerevan, Armenia
}

August 11, 2018

\begin{abstract}
Some characteristic features in the radiation from a relativistic electron uniformly rotating along an equatorial orbit around a dielectric ball have been studied. It was shown that at some harmonics, in case of weak absorption of radiation in the ball material, the electron may generate radiation field quanta exceeding in several dozens of times those generated by electron rotating in a continuous, infinite and transparent medium having the same real part of permittivity as the ball material. The rise of high power radiation is due to the fact that electromagnetic oscillations of Cherenkov radiation induced along the trajectory of particle are partially locked inside the ball and superimposed in nondestructive way.
\end{abstract}

PACS: 41.60.Bq; 41.60Ap

Keywords: Relativistic electron; Cherenkov radiation; Synchrotron radiation

\section{Introduction}

Due to such unique properties as the high intensity, high degree of collimation, and wide spectral range (see [1]- [6] and references therein) Synchrotron Radiation (SR) serves as an extraordinary research tool for advanced studies in both the fundamental and applied sciences. Nowadays it is used worldwide by thousands of scientists in many disciplines like physics, chemistry, material science and structural biology. These applications motivate the importance of analyzing various mechanisms for control of SR parameters. From this point of view it is of interest to study the influence of medium on spectral and angular distributions of SR. Investigations of this kind are also important for a number of astrophysical problems [4, 7].

The characteristics of high-energy electromagnetic processes in the presence of material are essentially changed by giving rise to new types of phenomena, the well-known examples of which are Cherenkov [8]-[10] and diffraction radiation. The operation of many devices intended for production of electromagnetic radiation is based on interactions of relativistic electrons with matter (see, e.g., 11]).

As was shown in 12], where SR from a charged particle rotating in a homogeneous medium was considered (see also [10, 13]), the interference between SR and Cherenkov Radiation (CR) had interesting consequences. New interesting phenomena occur in case of inhomogeneous media. A well-known example here is the transition radiation. In particular, the interfaces between media can be used for monitoring the flows of radiation emitted in various systems. In a series

\footnotetext{
*E-mail address: levonshg@web.am

${ }^{\dagger}$ E-mail address: arzumsr@yandex.ru
} 
of papers initiated in [14-[16] it was shown that the interference between SR and CR induced at boundaries of spherical or cylindrical configuration leads to interesting effects.

Investigations of radiation from a charge rotating along an equatorial orbit about/inside a dielectric ball showed [17, 18] that when the Cherenkov condition for the ball material and particle speed is satisfied, there appear high narrow peaks in the spectral distribution of the number of quanta emitted to outer space at some specific values of the ratio of ball-to-particle orbit radii. In the vicinity of these peaks the radiated energy exceeds the corresponding value for the case of homogeneous and unbounded medium by several orders of magnitude. However in [17, 18, the phenomena of absorption and dispersion of electromagnetic waves inside the ball material were not taken into account. The allowance for these phenomena was made in [19. In the present paper a new characteristic feature of such a high power radiation was revealed and a simple model that disclosed the physical cause of its generation was suggested.

It is worthwhile to point out that a similar phenomenon (less pronounced) takes place in the cylindrical symmetry case [20]-26]. E.g., the radiation emitted (i) from a longitudinal charged oscillator moving with constant drift velocity along the cylinder axis, and (ii) from a charged particle moving along a circle around a dielectric cylinder or (iii) along a helical orbit inside the cylinder are investigated in [20]-22], 23] and 24]-26] respectively. The latter type of motion is used in helical undulators for generating electromagnetic radiation in a narrow spectral interval. It is shown that under similar Cherenkov condition for permittivity of cylinder and the particle speed, high narrow peaks are present in the spectral-angular distribution for the number of radiated quanta. In the vicinity of these peaks the radiated energy exceeds the corresponding value for homogeneous medium case by several orders of magnitude.

The content of paper is organized as follows: in Section 2 the description of problem is given, and in Section 3 the method of solution is described and the final analytical expression for the intensity of radiation from a relativistic particle rotating about a ball is given. Numerical results are presented in Section 4. The characteristic features of phenomenon are discussed in Section 5 The cause of radiation amplification is established in Section 6. Similar processes of amplification typical for a chain of equidistant particles/bunches, are considered in the next section. In the last section the main results of paper are summarized.

\section{The description of problem}

Now consider the uniform rotation of relativistic electron in the equatorial plane of a dielectric ball in the magnetic field in empty space (see Fig. 11).

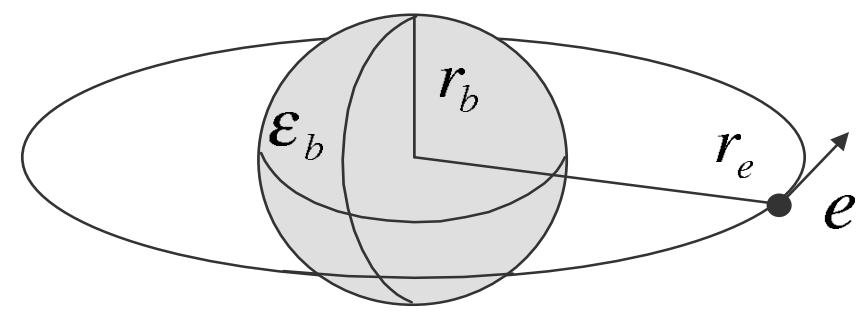

Figure 1: A relativistic electron rotating about a dielectric ball in its equatorial plane.

The permittivity of medium is the following step function of radial coordinate $\mathrm{r}$ :

$$
\varepsilon(r)=\varepsilon_{b}+\left(1-\varepsilon_{b}\right) \Theta\left(r-r_{b}\right),
$$

where $r_{b}$ is the radius of ball, and $\varepsilon_{b}=\varepsilon_{b}^{\prime}+i \varepsilon_{b}^{\prime \prime}$ is the complex valued permittivity of the ball material. We assume that the braking of electron due to the emission of radiation is compensated 
by an external influence (e.g., the electric force) that compels the particle to turn uniformly in a circle.

It is convenient to introduce the following dimensionless quantity:

$$
\mathrm{w}_{k T} / \hbar \omega_{k} \equiv n_{k}
$$

(the number of emitted quanta). Here $\mathrm{w}_{k T}$ is the energy radiated at frequency $\omega_{k}=k \omega_{0}$ during one period $T=2 \pi / \omega_{0}$ of electron gyration, $k=1 ; 2 ; 3 \ldots$ is the harmonic number, and $\hbar \omega_{k}$ is the energy of corresponding electromagnetic wave quantum.

It is known [10] that if the space as a whole is filled with a transparent substance (with constant $\varepsilon$ ) then

$$
n_{k}(\infty ; \mathrm{v}, \varepsilon)=\frac{n_{0}}{\beta \sqrt{\varepsilon}}\left[2 \beta^{2} J_{2 k}^{\prime}(2 k \beta)+\left(\beta^{2}-1\right) \int_{0}^{2 k \beta} J_{2 k}(x) d x\right],
$$

where $n_{0}=2 \pi e^{2} / \hbar c \cong 0.0459, \beta=\mathrm{v} \sqrt{\varepsilon} / c$ and $J_{k}(x)$ is the Bessel function of integer order. The case $\varepsilon=1$ of this formula corresponds to the synchrotron radiation in vacuum (see, e.g., [2, 3]).

We aimed at:

- calculation of $n_{k}=n_{k}$ (ball), when $\varepsilon(r)$ is the step function (II) and

- demonstration that in case of weak absorption of radiation in the ball material and at special choice of the values of $r_{b}$ and $\varepsilon_{b}$, the number of emitted quanta

$$
n_{k}\left(\mathrm{ball} ; \mathrm{v}, r_{b} / r_{e}, \varepsilon_{b}\right) \gg n_{k}\left(\infty ; \mathrm{v}, \varepsilon_{b}^{\prime}\right) \text {. }
$$

\section{The final formula}

We have obtained [14, 15, 17] (see also [18]) the following expression

$$
n_{k}\left(\text { ball; } \mathrm{v}, x, \varepsilon_{b}\right)=\frac{2 n_{0}}{k} \sum_{s=0}^{\infty}\left(\left|a_{k E}(s)\right|^{2}+\left|a_{k H}(s)\right|^{2}\right)
$$

for the number of quanta emitted by the electron during one revolution. Here $x \equiv r_{b} / r_{e}<1$ and

$$
\begin{aligned}
& a_{k E}(s)=k b_{l}(E) P_{l}^{k}(0) \sqrt{(l-k) ! / l(l+1)(2 l+1)(l+k) !}, \quad l=k+2 s, \\
& a_{k H}(s)=\left.b_{l}(H) \sqrt{\frac{(2 l+1)(l-k) !}{l(l+1)(l+k) !}} \frac{d P_{l}^{k}(y)}{d y}\right|_{y=0}, \quad l=k+2 s+1
\end{aligned}
$$

are dimensionless amplitudes describing the contributions of electrical $(E)$ and magnetic $(H)$ type multipoles respectively, $P_{l}^{k}(y)$ are the associated Legendre polynomials, and $b_{l}(E), b_{l}(H)$ are the following factors depending on $k, \mathrm{v}, x$ and $\varepsilon_{b}$ :

$$
\begin{aligned}
b_{l}(H)= & i u\left[j_{l}(u)-h_{l}(u) \frac{\left\{j_{l}\left(x u_{b}\right), j_{l}(x u)\right\}_{x}}{\left\{j_{l}\left(x u_{b}\right), h_{l}(x u)\right\}_{x}}\right], \quad u=k \mathrm{v} / c, \quad u_{b}=k \mathrm{v} \sqrt{\varepsilon_{b}} / c \\
b_{l}(E)= & (l+1) b_{l-1}(H)-l b_{l+1}(H)+x^{-2}\left(1-\varepsilon_{b}\right)\left[j_{l-1}\left(x u_{b}\right)+j_{l+1}\right. \\
& \left.\times\left[u_{b}\right)\right] \times \\
& \times\left[h_{l-1}(u)+h_{\underline{l+1}}(u)\right] \frac{l(l+1) u_{b} j_{l}\left(x u_{b}\right)}{l z_{l-1}^{l}+(l+1) z_{l+1}^{l}} .
\end{aligned}
$$


Here $h_{l}(y)=j_{l}(y)+i \mathrm{n}_{l}(y)$, and $j_{l}(y), \mathrm{n}_{l}(y)$ are spherical Bessel and Neumann functions respectively. In (6) we used the following notations:

$$
\begin{aligned}
\{a(x \alpha), b(x \beta)\}_{x} & \equiv a \frac{\partial b}{\partial x}-\frac{\partial a}{\partial x} b \\
f_{\underline{l}}(x) & \equiv f_{l}(x) /\left\{j_{l}\left(x u_{b}\right), h_{l}(x u)\right\}_{x} \\
z_{\nu}^{l} & \equiv \frac{u j_{\nu}\left(x u_{b}\right) h_{l}(x u) \varepsilon_{b}-u_{b} j_{l}\left(x u_{b}\right) h_{\nu}(x u)}{u j_{\nu}\left(x u_{b}\right) h_{l}(x u)-u_{b} j_{l}\left(x u_{b}\right) h_{\nu}(x u)} .
\end{aligned}
$$

In the absence of dielectric ball $\left(\varepsilon_{b}=1\right)$ the calculations by means of our formula (4) give the same results as those obtained using the well-known synchrotron radiation theory formula (3).

\section{The results of numerical calculations}

Now consider the radiation generated by the electron at some harmonic $\omega_{k}=k \omega_{0}$, e.g., for $k=8$. In Fig 2 the number $n_{8}(x)$ of emitted quanta was plotted versus the ratio $x=r_{b} / r_{e}$ of

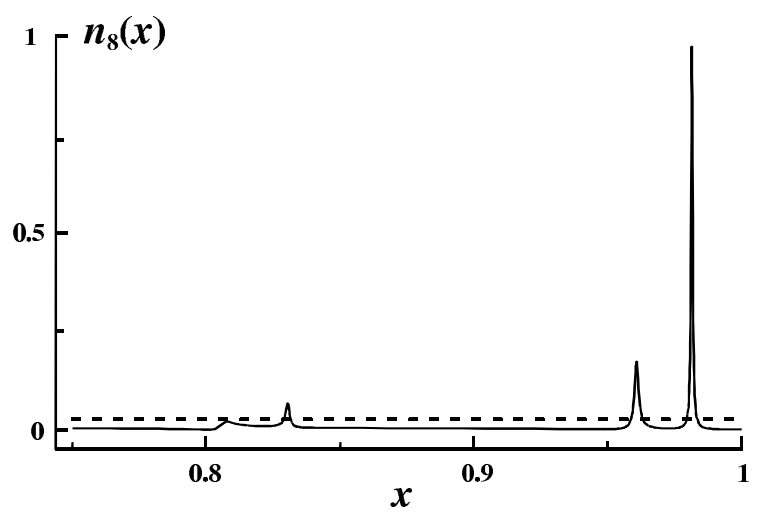

Figure 2: The number $n_{k}(x)$ of electromagnetic field quanta generated per revolution of electron about a dielectric ball depending on the ratio $x=r_{b} / r_{e}$ of the radius of ball and that of electron orbit. The harmonic number $k=8$, the electron energy $E_{e}=2 \mathrm{MeV}$, the radius of its orbit $r_{e}=3.69 \mathrm{~cm}$. The corresponding radiation is at the wavelength $3 \mathrm{~cm}$ (in vacuum). The ball is made of melted quartz. The dielectric losses of energy inside the ball material are taken into account. For explanation see the text.

ball to electron orbit radii. The energy and the orbit radius of the particle were taken to be $E_{e}=2 \mathrm{MeV}$ and $r_{e}=3.69 \mathrm{~cm}$ respectively. Here corresponding to the 8 th harmonic is the radiation at frequency $\omega_{8} / 2 \pi=10^{10} \mathrm{~Hz}$ and wavelength $\lambda_{8}=3 \mathrm{~cm}$ (in vacuum). The number of quanta was calculated in [19] by formula (4) with due regard for the dispersion and dielectric losses of energy inside the substance of ball under the assumption that the ball is made of melted quartz, the value of permittivity of which is [27, 28]:

$$
\varepsilon_{b}\left(\omega_{8}\right)=\varepsilon_{b}^{\prime}+i \varepsilon_{b}^{\prime \prime}=3.78(1+0.0001 i)
$$

According to (3) the number of quanta emitted on the 8th harmonic by electron with above values of $E_{e}$ and $r_{e}$ at rotation in a continuous, infinite and transparent medium with $\varepsilon=3.78$ would be 10

$$
n_{8}(\infty) \cong 0.0274
$$


(the horizontal dashed line in Fig 22). If the particle did not rotate, but moved rectilinearly in the same medium with energy $E_{e}=2 \mathrm{MeV}$, then during the period of time $T=2 \pi / \omega_{0}$ it would have emitted in a narrow frequency band $\Delta \omega=\omega_{0}$

$$
n_{\Delta \omega}(\infty)=(\mathrm{v} / c-c / \mathrm{v} \varepsilon) n_{0} \cong 0.0318
$$

quanta (see, e.g., [8]-10]). It is evident that $n_{\Delta \omega}(\infty) \sim n_{8}(\infty)$. In the absence of ball (synchrotron radiation [1]- 6 ])

$$
n_{8}(\mathrm{vac}) \cong 0.00475 \text {. }
$$

According to Fig 2 $n_{8}(x) \sim n_{8}$ (vac) practically for all $x$ except for $0.8<x<0.85$ and $0.95<x<1$. There are peaks in these ranges and for the highest one

$$
x^{*}=0.9815 \text { and } n_{8}\left(\text { ball } ; x^{*}\right) \cong 0.951 \quad \Longrightarrow \quad n_{8}\left(\text { ball } ; x^{*}\right) / n_{8}(\infty) \cong 35 .
$$

Here more than 30 -fold magnification of $n_{8}$ (from $\approx 0.03$ to $\approx 1.0$ ) is due to the forced uniform rotation of electron around the ball under the action of an external energy source. The corresponding value of ball radius $r_{b}=3.62 \mathrm{~cm}$ and, consequently, the distance $r_{e}-r_{b}$ between the rotating electron and the ball surface should be $0.7 \mathrm{~mm}$. Apparently,

$$
n_{8}(\operatorname{vac})<<n_{\Delta \omega}(\infty) \sim n_{8}(\infty)<<n_{8}\left(\text { ball; } x^{*}\right) .
$$

Similar results may be obtained for a series of other values of $k>>1$, as well as for electrons with $1 \leq E_{e} \leq 5 \mathrm{MeV}$ energy and balls with $1 \leq \varepsilon_{b}^{\prime} \leq 5$ and $\varepsilon_{b}^{\prime \prime} / \varepsilon_{b}^{\prime}<<1$.

\section{Peculiarities of the phenomenon}

It is significant that the condition

$$
n_{k}\left(\text { ball; } \mathrm{v}, x^{*}, \varepsilon_{b}\right)>>n_{k}\left(\infty ; \mathrm{v}, \varepsilon_{b}^{\prime}\right)
$$

holds

(A) only for a number of values of $r_{b} / r_{e}=x^{*}$ and also

(B) when simultaneously the Cherenkov condition

$$
\mathrm{V}_{*} \sqrt{\varepsilon_{b}^{\prime}} / c>1
$$

for speed $\mathrm{v}_{*} \equiv x^{*} \mathrm{v}=r_{b} \omega_{0}$ of particle "image" on the ball surface (see. Fig 3) and the real part $\varepsilon_{b}^{\prime}$ of the permittivity of ball material is satisfied.

For all peaks (14) the characteristic propagation lengths $l=2 c \sqrt{\varepsilon_{b}^{\prime}} / \omega_{k} \varepsilon_{b}^{\prime \prime}$, after passage of which the amplitude of electromagnetic oscillations decreases in $e$ times, are always many times greater than the ball radius:

$$
l>r_{b} .
$$

For this reason the third condition may be formulated as:

(C) inside the ball the electromagnetic oscillations may pass the distances that exceed many times the radius $r_{b}$ of ball (before oscillations are absorbed or leave the boundaries of ball). 


\section{The reason of radiation amplification}

If the electron is relativistic, then in addition to the synchrotron radiation it may also generate Cherenkov radiation. Its rise is attributed to the fact that the field coupled with the relativistic electron partially penetrates the ball and rotates together with the particle. At small distances from the surface of ball: $r_{e} \approx r_{b}$, the speed of this field displacement inside the ball may be

larger than the phase speed $c / \sqrt{\varepsilon_{b}^{\prime}}$ of light in the ball material, and then CR with wavelengths [8] -10 ]

$$
\lambda_{k} / \sqrt{\varepsilon_{b}^{\prime}} \geq\left(r_{e}-r_{b}\right) .
$$

is generated inside the ball. In this section for visual explanation of numerical results given in Sections 45 we shall avail of the following simplified model.

Let us assume that at the moment $t_{A}$ the electron is in point $A$ of orbit and its image is in point $A^{*}$ (see. Fig 31). At this instant the electron generates CR along with the synchrotron

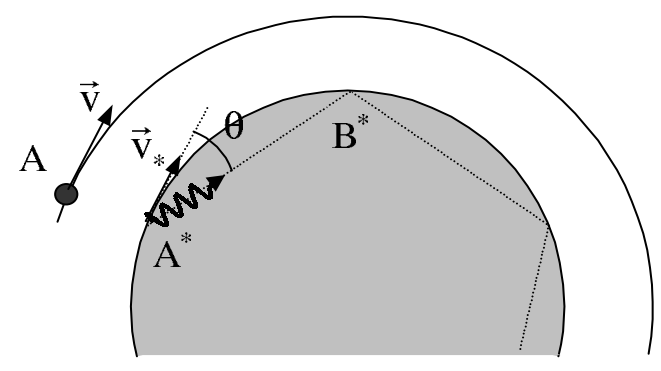

Figure 3: A rotating electron in point $A$ along with the synchrotron radiation generates a pulse of Cherenkov radiation (the wave line) in the vicinity of point $A^{*}$ inside the ball (the speed of electron field displacement inside the ball exceeds the phase speed of light in the ball material). The pulse travels to the vicinity of point $B^{*}$ and at least some part of radiation is subjected to an "almost total" internal reflection at $\theta$ angle.

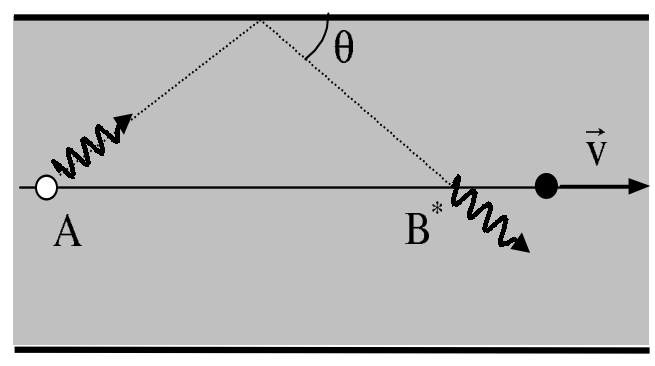

Figure 4: A relativistic charged particle moves along the axis of circular section waveguide filled with dielectric. The particle generates a pulse of Cherenkov radiation in the vicinity of point $A$ (the wave line). At its propagation the pulse at some instant crosses the trajectory of its faster-than-light source (axis of waveguide) in the vicinity of point $B^{*}$ at the same angle $\theta$, at which it was emitted. At that moment the pulse will fall behind the particle that will be found more to the right at some point $B$.

radiation, or to be more exact, generates CR pulse $W\left(A^{*} ; t \geq t_{A}\right)$ (the wavy line) in the vicinity of point $A^{*}$ inside the ball near the surface. The direction of $\mathrm{CR}$ is characterized by some 
radiation angle $\theta$. We shall use the following ansatz that $\theta$ is determined by the Cherenkov condition

$$
\cos \theta=c / \mathrm{v}_{*} \sqrt{\varepsilon_{b}^{\prime}}
$$

by analogy with the case of faster-than-light particle propagating rectilinearly in a continuous and infinite dielectric medium [8]-[10]. In (18) $\mathrm{v}_{*}=\mathrm{v} r_{b} / r_{e}$ is the speed of particle "image" on the surface of ball. According to such a simplified approach, the condition B of Section 5 establishes that the electron generates $\mathrm{CR}$ inside the dielectric ball. The pulse $W\left(A^{*} ; t \geq t_{A}\right)$ travels the distance $A^{*} B^{*}$ to the vicinity of point $B^{*}$ by the surface of ball and is partially absorbed by the ball material. In (18)

$$
\cos \theta>1 / \sqrt{\varepsilon_{b}^{\prime}}
$$

and, consequently, at least some part of radiation with the wavelength

$$
\lambda_{k} / \sqrt{\varepsilon_{b}^{\prime}}<<r_{b}
$$

(according to the laws of geometrical optics) will be subjected to an "almost total" internal reflection at $\theta$ angle. This process will continue till $W\left(A^{*} ; t \geq t_{A}\right)$ is totally absorbed and/or leaves the ball. At $\varepsilon_{b}^{\prime} / \varepsilon_{b}^{\prime \prime}>>1$ (small adsorption) the pulse $W\left(A^{*} ; t \geq t_{A}\right)$ "will have time" to reflect from the internal surface of ball

$$
m \approx \frac{l}{A^{*} B^{*}}=\frac{\varepsilon_{b}^{\prime} / \varepsilon_{b}^{\prime \prime}}{k \tan \theta}>>1
$$

times and, consequently, to travel the distance $m A^{*} B^{*} \sim m r_{b}>>r_{b}$ inside the ball in agreement with condition $\mathrm{C}$ of the previous section. Over the period of time $T_{l}=l \sqrt{\varepsilon_{b}^{\prime}} / c$, during which the oscillation amplitude in the pulse $W\left(A^{*} ; t \geq t_{A}\right)$ decreases in $e$ times, the electron will make

$$
N \equiv\left[T_{l} / T\right]=\left[\varepsilon_{b}^{\prime} / \pi k \varepsilon_{b}^{\prime \prime}\right]>>1
$$

revolutions along the orbit $([\tau]$ means the integer part of number $\tau)$. In this process the electron will appear in the vicinity of point $A$ many times at instants $t_{A}+T ; t_{A}+2 T ; \ldots t_{A}+N T$, and its image will appear in the vicinity of point $A^{*}$ as much times again and each time will generate CR pulse. All $N+1$ pulses $W\left(A^{*} ; t \geq t_{A}+s T\right)$ with $s=0 ; 1 ; 2 \ldots N$ will propagate inside the confined space (ball) along identical "trajectories". For the highest peak in Fig $2 m \approx 806$ and $N=398$.

The aforesaid is applicable to each point along the electron orbit. Being superimposed in the confined space (ball), the electromagnetic oscillations of numerous CR pulses will, naturally, damp each other. In this case the influence of ball will be small and the total radiation will not differ strongly from the synchrotron radiation. This is precisely the case for the most values of $x$ (see Fig 2). However, in some special cases the deviations from such a picture are possible. These take place in ranges $0.8<x<0.85$ and $0.95<x<1$ shown in Fig 2 Inside these ranges at some special choice of $x=r_{b} / r_{e} \equiv x^{*}$ (cf. with condition A in Section 5) the superposition of electromagnetic oscillations will be close to the in-phase one. In this case the total field may prove considerably more strong than that at $x \neq x^{*}$. The force that brakes the gyration of electron will also increase and the extra work of external force compelling the electron to rotate uniformly along the orbit will be spent for generation of more powerful $C R$. This is precisely the case at $x^{*}=0.9815$ in Fig 2 .

In Section 4 we determined the values of $x=x^{*}$ from the requirement of the presence of peak, $n_{k}\left(\right.$ ball; $\left.x^{*}\right)>>n_{k}(\infty)$, by numerical calculations by formula (4), disregarding (18) and neglecting the laws of geometrical optics. 
1. Thus, in general, the influence of ball on radiation from the particle is small and the total radiation differs slightly from synchrotron radiation.

2. However, at some special values of the ratio $r_{b} / r_{e} \equiv x^{*}$ high power CR will be generated.

3. Its rise is due to the fact that the electromagnetic oscillations of CR induced by the particle along all the trajectory are partially locked inside the ball and are superimposed in nondestructive way.

\section{Similar processes of amplification}

\subsection{Amplification of transition radiation $[28,29]$}

In 1996 and 1997 Prof. Wiedemann with co-workers experimentally observed a similar phenomenon for Transition Radiation (TR) from a chain of relativistic electron bunches of subpicosecond duration from the Stanford University Short Intense Electron source (SUNSHINE).

At the output of TR radiator (metallic foil) the radiation from one electron bunch was directed with the help of a special system of mirrors (influence of boundaries) back to the radiator so, that the radiation pulse arrived there at the instant of incidence of the other electron bunch to generate new TR pulse. So, a superposition of electromagnetic pulses emitted at different moments of time took place within the formation zone of radiation.

In the case of ball, the superposition of electromagnetic pulses emitted at different moments of time also takes place in the radiation zone. However, the pulses are emitted by the same particle (not different particles).

\subsection{Amplification of Cherenkov radiation inside a waveguide}

Let us consider CR from a chain of equidistant charges moving along the axis of circular section waveguide filled with transparent and nondispersive dielectric (e.g.., teflon in the range of wavelengths $\lambda>1 \mathrm{~mm}$ [27, 28]).

In Fig 4 the first charge is shown to be in point $A$. Let us trace the propagation of CR pulse $W_{1}\left(A ; t \geq t_{A}\right)$ induced by the first charge and emitted in small vicinity of point $A$ at the instant $t_{A}$ (wave line in Fig (4). At its propagation the pulse $W_{1}\left(A ; t \geq t_{A}\right)$ at some instant $t_{B}$ will cross the trajectory of charges (axis of waveguide) in the vicinity of some point $B^{*}$ at the same angle $\theta$, at which it was emitted. At that moment $W_{1}\left(A ; t \geq t_{A}\right)$ will fall behind its faster-than-light source that will be found more to the right at some point $B$.

The second charge of the chain is more to the left at the distance of $d$ from the first one and if $d \cong B^{*} B$ it will cross the vicinity of point $B^{*}$ simultaneously with the pulse $W_{1}\left(A ; t \geq t_{A}\right)$. CR pulse $W_{2}\left(B^{*} ; t \geq t_{B}\right)$ generated by the second charge in the vicinity of point $B^{*}$ will be formed and simultaneously interfere with $W_{1}\left(A ; t \geq t_{A}\right)$ for all $t \geq t_{B}$. If the direction of CR $\cos \theta=c / \mathrm{v} \sqrt{\varepsilon}$, and the radius $a$ of waveguide are known, one can easily calculate the distance

$$
B^{*} B=2 a \tan \theta
$$

of a pulse $W_{1}\left(A ; t \geq t_{A}\right)$ from its source at the instant $t_{B}$. It is clear that at $d \cong B^{*} B=2 a \tan \theta$ the generation of pulses in regions adjacent to the second, third and subsequent charges will take place simultaneously with their interference with the pulses emitted earlier by charges propagating ahead of them. Note, that a similar process may take place for all $d$ multiple to the length of $B^{*} B$ and, in particular, at $x \equiv d / B^{*} B \cong 1 ; 2 ; 3 \ldots$.

The superposition of pulses may be accompanied by their suppression or amplification depending on this or that value of the phase difference. For some values of $x \equiv x^{*}$ the in-phase superimposition of oscillations is possible. 
Analytical and numerical calculations carried out in 31] have shown that the in-phase superposition takes place if

- $x^{*} \cong 1$ (the case of power quasi-monochromatic CR) and

- $x^{*}=4$ (powerful CR in wider frequency band).

In both the cases the pulses are superimposed in-phase in regions directly adjacent to the radiating charges. But for all that the superimposed pulses are generated by different charges. In case of ball the pulses are emitted by the same particle.

\section{Conclusions}

1) We have studied the radiation from a relativistic electron at uniform rotation about a dielectric ball with due regard for dielectric losses of energy inside the ball material. Here, in addition to the synchrotron radiation the electron may also emit Cherenkov radiation. Its rise is attributed to the fact that the field coupled with the relativistic electron partially penetrates the ball and rotates together with the particle. At small distances from the surface of ball: $r_{e} \approx r_{b}$, the speed of this field displacement may be larger than the phase speed of light in the ball material and then Cherenkov radiation with wavelengths (17) is emitted inside the ball. The peculiarities in total radiation at different harmonics $\omega_{k}$ due to the influence of matter and ball radius are investigated theoretically.

2) In general, the influence of ball on radiation from the particle is small and radiation differs slightly from synchrotron radiation. However, in case of weak absorption $\left(\varepsilon_{b}^{\prime \prime}<<\varepsilon_{b}^{\prime}\right)$ in the ball material at some harmonics with $k>>1$ the electron may generate $n_{k} \geq 1$ quanta of electromagnetic field during one rotation period. E.g., an electron of $2 \mathrm{MeV}$ energy uniformly rotating at the distance of $0.7 \mathrm{~mm}$ from the surface of fused quartz ball with radius $r_{b}=3.62 \mathrm{~cm}$ will generate approximately one quantum $\left(n_{8} \approx 1.0\right)$ of electromagnetic field at the wavelength $\lambda_{8} \approx 3 \mathrm{~cm}$ (8th harmonic) during one revolution (see the highest peak in Fig 22). This value is more than 30 -fold greater than the similar value of $n_{8}$ for electron rotating in a continuous, infinite and transparent medium having the same real part $\varepsilon^{\prime}$ of permittivity as that for the ball (see (9) and the dashed line in Fig 22).

3) Such a magnification of $n_{k}$ is due to the presence of external source of energy stimulating a uniform rotation of electron around the ball. It is possible only if

- the ratio between the radius of ball and that of electron orbit takes on a number of fixed values (see, e.g., (12))

- the Cherenkov condition (15) for the speed of particle "image" on the ball surface and the ball material is satisfied and

- inside the ball the Cherenkov radiation field passes the distances that are many times greater than the radius $r_{b}$ of ball (before the radiation will be absorbed or leave the ball boundaries).

4) High power radiation rises due to the fact that electromagnetic oscillations of Cherenkov radiation induced by the particle along all its trajectory are partially locked inside the ball and are superimposed in nondestructive way (Section [6).

\section{Acknowledgements}

The authors are thankful to Professor A.R. Mkrtchyan for general encouragement and A.A. Saharian, A.S. Kotanjyan for stimulating discussions. One of the authors (S.R.A.) is especially thankful to S. Bellucci for attention and assistance. 
The work is supported by Ministry of Education and Science of RA (Grant No. 0063).

\section{References}

[1] E-E Koch (ed.), Handbook on Synchrotron Radiation, North Holland, Amsterdam, 1983.

[2] I.M. Ternov, V.V. Mikhailin, V.R. Khalilov, Synchrotron Radiation and Its Applications, Harwood Academic, Amsterdam, 1985.

[3] A.A. Sokolov, I.M. Ternov, Radiation from Relativistic Electron, ATP, New York, 1986.

[4] V.A. Bordovitsyn (ed.), Synchrotron Radiation Theory and Its Development, World Scientific, Singapore, 1999.

[5] H. Hiedemann, Synchrotron Radiation, Springer-Verlag, Germany, 2003.

[6] A. Hofman, The Physics of Synchrotron Radiation, Cambridge University Press, Cambridge, 2004.

[7] G.B. Rybicky, A.P. Lightman, Radiative Processes in Astrophysics, J. Wiley, New York, 1979.

[8] J.V. Jelley, Cherenkov Radiation and Its Applications, Pergamon Press, London, 1958.

[9] B.M. Bolotovskii, Physics-Uspekhi 75 (1961) 295.

[10] V.P. Zrelov, Vavilov-Cherenkov Radiation (and Its Applications in High Energy Physics), Atomizdat, Moscow, 1968 (in Russian).

[11] P. Rullhusen, X. Artru, P. Dhez, Novel Radiation Sources Using Relativistic Electrons, World Scientific, Singapore, 1998.

[12] V.N. Tsytovich, Westnik MGU 11 (1951) 27 (in Russian).

[13] K. Kitao, Prog. Theor. Phys. 23 (1960) 759.

[14] S.R. Arzumanyan, L.Sh. Grigoryan, A.A. Saharian, Izv. Nats. Akad. Nauk Arm., Fiz. (Engl. Transl.: J. Contemp. Phys.) 30 (1995) 99.

[15] S.R. Arzumanyan, L.Sh. Grigoryan, A.A. Saharian, Kh.V. Kotanjian, Izv. Nats. Akad. Nauk Arm., Fiz. (Engl. Transl.: J. Contemp. Phys.) 30 (1995) 106.

[16] L.Sh. Grigoryan, A.S. Kotanjyan, A.A. Saharian, Izv. Nats. Akad. Nauk Arm., Fiz. (Engl. Transl.: J. Contemp. Phys.) 30 (1995) 239.

[17] L.Sh. Grigoryan, H.F. Khachatryan, S.R. Arzumanyan, Izv. Nats. Akad. Nauk Arm., Fiz. (Engl. Transl.: J. Contemp. Phys.) 33 (1998) 267 (Preprint cond-mat/0001322).

[18] L.Sh. Grigoryan, H.F. Khachatryan, S.R. Arzumanyan, Izv. Nats. Akad. Nauk Arm., Fiz. (Engl. Transl.: J. Contemp. Phys.) 37 (2002) 327.

[19] M.L. Grigoryan, V National Conference on Application of the X-rays, Synchrotron Radiation, Neutrons, and Electrons for Studying NANO-Materials (RSNE NANO-2005), 14-19 November 2005, Moscow, Book of Abstracts, p.302 (in Russian); preprint hep-th/0512080. 
[20] A.S. Kotanjyan, A.A. Saharian, Izv. Nats. Akad. Nauk Arm., Fiz. (Engl. Transl.: J. Contemp. Phys.) 37 (2002) 135.

[21] A.A. Saharian, A.S. Kotanjyan, Izv. Nats. Akad. Nauk Arm., Fiz. (Engl. Transl.: J. Contemp. Phys.) 38 (2003) 288.

[22] A.A. Saharian, A.S. Kotanjyan, Nucl. Instr. and Meth. B 226 (2004) 351.

[23] A.S. Kotanjyan, H.F. Khachtaryan, A.V. Petrosyan, A.A. Saharian, Izv. Nats. Akad. Nauk Arm., Fiz. (Engl. Transl.: J. Contemp. Phys.) 35 (2000) 115.

[24] A.S. Kotanjyan, A.A. Saharian, Izv. Nats. Akad. Nauk Arm., Fiz. (Engl. Transl.: J. Contemp. Phys.) 36 (2001) 310; ibid 37 (2002) 263; Mod. Phys. Lett. A 17 (2002) 1323.

[25] A.S. Kotanjyan, Nucl. Instr. and Meth. B 201 (2003) 3.

[26] A.A. Saharian, A.S. Kotanjyan, J. Phys. A 38 (2005) 4275.

[27] G.C. Southworth, Principles and Applications of Waveguide Transmission, New York, 1950.

[28] E.M. Voronkova, B.N. Grechushnkiov, G.I. Distler, I.P. Petrov, Optical Materials for Infrared Technology, Nauka, Moscow, 1965 (in Russian).

[29] H.C. Lihn, D. Bocek, M. Hernandez, P. Kung, C. Settakorn, H. Wiedemann, Phys. Rev. Lett. 76 (1996) 4163.

[30] C. Settakorn, M. Hernandez, H. Wiedemann, Stimulated Transition Radiation in the FarInfrared, SLAC-PUB-7587, August 1997.

[31] L.Sh. Grigoryan, H.F. Khachatryan, A.A. Saharian, Kh.V. Kotanjyan, S.R. Arzumanyan, M.L. Grigoryan, Izv. Nats. Akad. Nauk Arm., Fiz. (Engl. Transl.: J. Contemp. Phys.) 40 (2005) 155. 